\title{
Ultrafast Excited-State Deactivation of the Bacterial Pigment Violacein
}

Published as part of The Journal of Physical Chemistry virtual special issue "Veronica Vaida Festschrift". Ashley A. Beckstead, ${ }^{\dagger}$ Yuyuan Zhang, ${ }^{\ddagger}$ Jonathan K. Hilmer, ${ }^{\dagger}, \perp$ Heidi J. Smith, ${ }^{\S}$ Emily Bermel, ${ }^{\S}, \#$ Christine M. Foreman, $*, \&, \|$ and Bern Kohler*, ${ }^{\dagger}, \ddagger$

${ }^{\ddagger}$ Department of Chemistry and Biochemistry, The Ohio State University, 100 West 18th Avenue, Columbus, Ohio 43210, United States

${ }^{\dagger}$ Department of Chemistry and Biochemistry, ${ }^{\S}$ Center for Biofilm Engineering, and ${ }^{~}$ Chemical and Biological Engineering Department, Montana State University, Bozeman, Montana 59717, United States

Supporting Information

ABSTRACT: The photophysical properties of the natural pigment violacein extracted from an Antarctic organism adapted to high exposure levels of UV radiation were measured in a combined steady-state and time-resolved spectroscopic study for the first time. In the low-viscosity solvents methanol and acetone, violacein exhibits low fluorescence quantum yields on the order of $1 \times 10^{-4}$, and femtosecond transient absorption measurements reveal excited-state lifetimes of $3.2 \pm 0.2$ and $4.5 \pm 0.2 \mathrm{ps}$ in methanol and acetone, respectively. As solvent viscosity is increased, both the fluorescence quantum yield and excited-state lifetime of this intensely colored pigment increase dramatically, and stimulated emission decays 30-fold more slowly in glycerol than in methanol at room temperature. Excited-state deactivation is suggested to occur via a molecular-rotor mechanism in which torsion about an interring bond leads to a conical intersection with the ground state.

\section{INTRODUCTION}

Ultraviolet (UV) radiation from the sun is harmful to living organisms because of photochemical damage to critical biomolecules that can lead to mutation and cell death. Sunexposed organisms must therefore adopt strategies to avoid photodamage. Some phototrophic microorganisms produce UV-screening molecules as a defense mechanism against solar irradiation. ${ }^{1}$ For example, high solar irradiances induce the production of UV-absorbing compounds such as scytonemin in cyanobacteria $^{2,3}$ and mycosporine-like amino acids in coral algae and phytoplankton. ${ }^{4,5}$ In addition, it has been shown that organisms equipped with carotenoid pigments are more resistant to photodamage. ${ }^{6}$

Bacteria in Antarctica particularly benefit from photoprotective pigments due to the presence of continuous sunlight through the Austral summer and increased UV radiation due to ozone depletion in the polar region. ${ }^{7,8}$ Supraglacial streams are short-lived and seasonally subject to continuous solar irradiation. Studying bacterial populations in the harsh environmental conditions of supraglacial streams provides insight into the requirements necessary for survival at life's limits and potentially extraterrestrially. ${ }^{9}$ Janthinobacterium sp. CG23_2, an aerobic psychrotolerant, rod-shaped organism isolated from the Cotton Glacier supraglacial stream in the Antarctic Dry Valleys ( $\left.77^{\circ} 07 \mathrm{~S}, 161^{\circ} 50 \mathrm{E}\right)$, produces the bisindole, purple pigment violacein. ${ }^{10}$ This pigment has a structure consisting of two indole rings (Figure 1) that is uncommon among natural pigments. ${ }^{11}$<smiles>O=C1NC(c2c[nH]c3ccc(O)cc23)=CC1=C1C(=O)Nc2ccccc21</smiles>

Violacein

Figure 1. Chemical structure of violacein. The compound consists of (from left to right) 5-hydroxyindole, 2-pyrrolidone, and oxindole heterocycles.

Violacein production increases in response to UV irradiation in a closely related Antarctic bacterium, resulting in increased survival. ${ }^{12}$ This protection could arise from the antioxidative properties of violacein, ${ }^{13,14}$ which could mitigate cellular oxidative stress by scavenging reactive oxygen species generated by UV radiation. In addition, violacein absorbs strongly across

Received: June 12, 2017

Revised: July 19, 2017

Published: July 28, 2017 
the UV $\left(\varepsilon=1.6 \times 10^{3} \mathrm{M}^{-1} \mathrm{~cm}^{-1}\right.$ at $\left.\lambda=310 \mathrm{~nm}\right)$ and visible $(\varepsilon$ $=3.4 \times 10^{3} \mathrm{M}^{-1} \mathrm{~cm}^{-1}$ at $\left.\lambda=570 \mathrm{~nm}\right)$ spectral regions due to its highly conjugated molecular structure, which consists of joined 5-hydroxyindole (5-HI), 2-pyrrolidone (2-PYR), and oxindole (OX) ring systems (Figure 1). The latter two moieties are connected by a double bond contributing to the pigment's overall conjugation. The strong absorption across a broad spectral region suggests that violacein may also function as a sunscreen.

Molecular sunscreens absorb sunlight strongly (i.e., have high molar extinction coefficients at UV and visible wavelengths) and are photostable. ${ }^{15}$ Molecular photostability is typically the result of ultrashort (hundreds of femtoseconds to tens of picoseconds) excited-state lifetimes and complete recovery of the ground-state population. ${ }^{16,17}$ This occurs when the molecule undergoes ultrafast internal conversion (UIC) to the ground state, a hallmark of molecular photostability. ${ }^{18}$ Here, we study the photophysics of violacein for the first time to understand its potential as a sunscreen. Femtosecond transient absorption (TA) measurements of violacein isolated from Janthinobacterium sp. CG23_2 show that the pigment has an excited-state lifetime of just $\overline{3}$ ps in methanol, consistent with its low fluorescence quantum yield. We observe that both the excited-state lifetime and fluorescence quantum yield increase dramatically with increasing solvent viscosity, indicating that the rate of deactivation is dependent on the solvent environment.

\section{EXPERIMENTAL METHODS}

The supraglacial streamwater samples were collected from the Cotton Glacier, Antarctica. ${ }^{10}$ Janthinobacterium sp. CG23_2 was grown to mid-exponential phase in the dark in a generalrecovery low-nutrient media, R2A (Difco). Samples were concentrated via centrifugation (10000 g for $15 \mathrm{~min})$. The supernatant, insoluble in aqueous solution, was removed and replaced with an equal volume of methanol (99.8\% Sigma) to extract violacein. Samples were incubated for $12 \mathrm{~h}$ at $4{ }^{\circ} \mathrm{C}$ and centrifuged $(10000 \mathrm{~g}$ for $15 \mathrm{~min})$ again to separate the extracted pigment from cell debris. Although solvent extraction separated the pigment from most of the cell components, the crude pigment extract solutions were further processed using solid-phase extraction purification, as described in the Supporting Information. The identity of the molecule was confirmed by the mass-to-charge ratio obtained from electrospray ionization mass spectrometry (see Figure $S 1$ and associated text).

UV-vis absorption spectra were recorded using a UVvisible spectrophotometer (Lambda 25, PerkinElmer) with solutions held in a $1 \mathrm{~mm}$ path length quartz cuvette (Starna). Steady-state emission spectra were measured at room temperature $\left(20 \pm 2{ }^{\circ} \mathrm{C}\right)$ using a commercial fluorometer (Fluorolog, HORIBA Jobin Yvon). Samples for emission measurements were held in a $1 \mathrm{~cm}$ path length quartz cuvette (Starna), and solution concentrations were adjusted to an absorbance of 0.2 to avoid reabsorption of emitted light. The spectra were corrected for the spectral response of the instrumentation and for solvent impurity emission by subtracting solvent-only background spectra. All spectra were collected after $600 \mathrm{~nm}$ excitation using $3.0 \mathrm{~nm}$ bandpass slit widths, $2.5 \mathrm{~nm}$ sampling, and $20.0 \mathrm{~s}$ integration time.

The pump-probe TA instrumentation has been described previously, ${ }^{19}$ but we briefly summarize the pertinent details here. The $800 \mathrm{~nm}$ laser fundamental was produced by a $1 \mathrm{kHz}$
Ti:sapphire regenerative amplifier (Libra-HE, Coherent). Pump and probe pulses were generated using two separate optical parametric amplifiers (OPerA Solo, Coherent), which were pumped by $\sim 1 \mathrm{~W}$ of the fundamental. The $600 \mathrm{~nm}$ pump was attenuated to $0.9 \mu \mathrm{J}$ and focused to a spot size of $\sim 670 \mu \mathrm{m}$ (full width at half-maximum (fwhm)). The $265 \mathrm{~nm}$ pump energy was also attenuated to $0.9 \mu \mathrm{J}$ with a spot size of $\sim 300 \mu \mathrm{m}$ (fwhm). Probe pulses at 720 and $570 \mathrm{~nm}$ were used to interrogate stimulated emission and ground-state bleach kinetics, respectively (see Discussion for further details). Pump-probe delay time was controlled by a motorized translation stage that provides up to 3 ns of delay. A mechanical chopper (New Focus) operating at $333 \mathrm{~Hz}$ let through every third pump pulse. The linearly polarized pump and probe pulses were adjusted to the magic angle $\left(54.7^{\circ}\right)$ with respect to one another to minimize signal contributions from molecular reorientation. Sample solutions were held within a stationary 1 $\mathrm{mm}$ path length quartz cuvette during TA measurements. Kinetic signals were fit using a sum of exponentials in the Igor Pro 6.36 (WaveMetrics Inc.) program, as described previously. ${ }^{20}$ All stated uncertainties are twice the standard error estimated by the fitting program.

\section{RESULTS}

3.1. Excited-State Deactivation in Methanol. The UVvisible absorption spectrum of violacein in methanol is shown in Figure 2a. Consistent with the spectrum of purified violacein in ethanol reported previously, ${ }^{21}$ our violacein sample exhibits a strong long-wavelength absorption band with a maximum at $569 \mathrm{~nm}\left(\lambda_{\max }^{\text {abs }}\right)$. On the basis of semiempirical calculations of violacein, Dias et al. assigned this band to $\mathrm{a}^{1} \pi \pi^{*}$ transition localized on the central 2-PYR ring, and they assigned a second band centered at $370 \mathrm{~nm}$ to charge transfer from the 5-HI to the central 2-PYR. ${ }^{22}$ We measured an extinction coefficient $(\varepsilon)$ of $3.4 \times 10^{3} \mathrm{M}^{-1} \mathrm{~cm}^{-1}$ at $570 \mathrm{~nm}$, which is comparable to the value of $3.7 \times 10^{3} \mathrm{M}^{-1} \mathrm{~cm}^{-1}$ at $570 \mathrm{~nm}$ in ethanol measured by Wang et al. $^{23}$ Mendes et al. reported a considerably higher value of $1.9 \times 10^{4} \mathrm{M}^{-1} \mathrm{~cm}^{-1}$ at $575 \mathrm{~nm}$ in ethanol, which may have been affected by incomplete pigment purification. ${ }^{24}$ As discussed in the Supporting Information, a single solvent extraction step is insufficient for purifying the pigment from cell components and results in greater absorbance than from a more pure solution (see Figure S2).

Figure $2 \mathrm{~b}$ displays the emission spectrum of violacein in methanol after $600 \mathrm{~nm}$ excitation. Maximum emission occurs near $680 \mathrm{~nm}$. It was reported earlier that maximum emission occurs at $675 \mathrm{~nm}$ in ethanol upon excitation at $575 \mathrm{~nm}$, but no details were provided. ${ }^{25}$ Using the laser dye LDS 751 in methanol as a standard, ${ }^{26}$ the fluorescence quantum yield of violacein was determined to be $1.4 \times 10^{-4}$.

TA measurements of violacein in methanol were collected using $600 \mathrm{~nm}$ excitation and 720 or $570 \mathrm{~nm}$ probing. The decay traces are shown in Figure 3. At both probe wavelengths, the negative $\Delta A$ signals recover monoexponentially and return completely to baseline with time constants of $\tau=3.2 \pm 0.2 \mathrm{ps}$ and $\tau=5.4 \pm 0.2$ ps for 720 and $570 \mathrm{~nm}$ probing, respectively. Identical TA kinetics were observed for the methanol solution using $265 \mathrm{~nm}$ pump/570 $\mathrm{nm}$ probe wavelengths (see Figure S5). UV-visible absorption spectra were collected before and after 600 laser exposure, and no measurable photodegradation or photoproduct formation was observed (Figure S6).

3.2. Emission and Ultrafast Spectroscopy in Viscous

Solvents. Violacein solutions were prepared in solvents of 

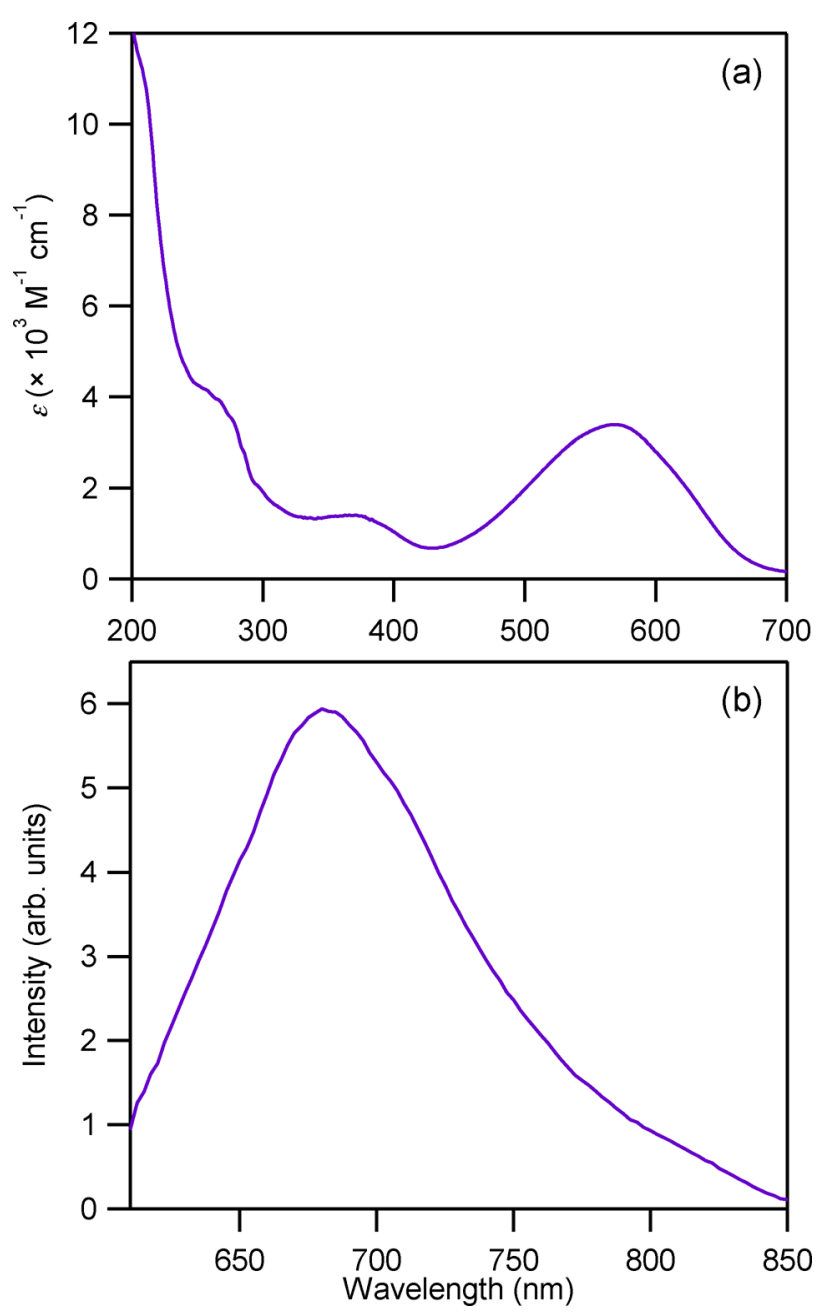

Figure 2. (a) UV-visible absorption and (b) steady-state fluorescence spectrum of violacein in methanol.

varying viscosity, including acetone, ethylene glycol, and glycerol. Ethylene glycol and glycerol have similar dielectric constants but differ considerably in viscosity (see Table 1$){ }^{27}$ The polar aprotic solvent acetone $(0.31 \mathrm{mPa} \cdot \mathrm{s})$ was used as a control, because it has a similar viscosity as methanol $(0.54$ $\mathrm{mPa} \cdot \mathrm{s}){ }^{27}$ All solvents also have similar refractive indices. ${ }^{27-29}$ All measurements in these solvents were performed at room temperature $\left(20 \pm 2{ }^{\circ} \mathrm{C}\right)$.

Steady-state absorption and fluorescence spectra are presented in Figure 4. The spectral characteristics of the steady-state UV-visible absorption and emission spectra are summarized in Table 2. Minor differences are observed in violacein absorption below $400 \mathrm{~nm}$, but in the visible region, the absorption maximum red shifts with increasing solvent polarity and viscosity. For $600 \mathrm{~nm}$ excitation, a solvatochromic shift of $\sim 12 \mathrm{~nm}$ is observed on going from the aprotic solvent acetone to the polar protic solvents, but little to no shifting of the emission band is observed as the solvent polarity increases within the series of protic solvents. Solvent viscosity, however, strongly affects the fluorescence quantum yield, which is $10 \times$ and $30 \times$ greater in ethylene glycol and glycerol, respectively, compared to the nonviscous methanol solvent (see Figure $4 \mathrm{~b}$ and Table 2).

The $720 \mathrm{~nm}$ decay kinetics of violacein in the different solvents (Figure 5a) reveal that the transient signals decay

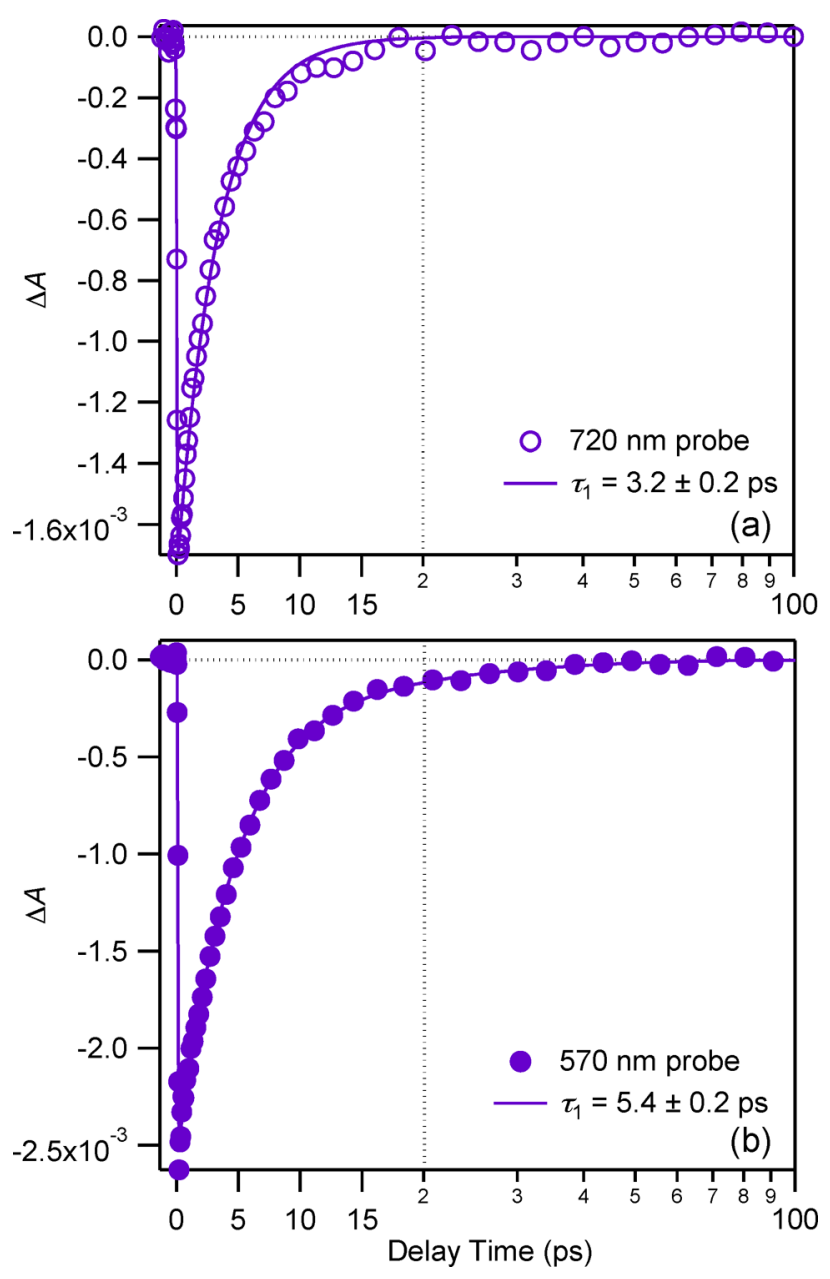

Figure 3. TA decay traces of violacein in methanol collected with 600 $\mathrm{nm}$ pump and (a) 720 and (b) $570 \mathrm{~nm}$ probe wavelengths. Both data sets were fit with single exponential functions using the time constants indicated in the figure legends. The vertical dashed lines in both panels indicate the break between linear and logarithmic scales of the $x$-axis.

Table 1. Physical Properties of the Different Solvents Used for Violacein Spectroscopic Measurements

$\begin{array}{cccc} & \text { viscosity }(\eta, \mathrm{mPa} \cdot \mathrm{s})^{a} & \begin{array}{c}\text { dielectric constant } \\ (\varepsilon)\end{array} & \begin{array}{c}\text { refractive index } \\ (n)\end{array} \\ \text { acetone } & 0.31 & 21.0 & 1.36^{a} \\ \text { methanol } & 0.54 & 33.0 & 1.33^{a} \\ \text { ethylene } & 16.1 & 41.4 & 1.43^{b} \\ \text { glycol } & & & \\ \text { glycerol } & 934 & 46.5 & 1.47^{c}\end{array}$

${ }^{a}$ Measured at $25{ }^{\circ} \mathrm{C}$, from ref $27 .{ }^{b}$ Measured at $25^{\circ} \mathrm{C}$, from ref 28 . ${ }^{c}$ Measured at $20^{\circ} \mathrm{C}$, from ref 29 .

much more slowly in the viscous solutions than in the lowviscosity solutions. The $600 \mathrm{~nm}$ pump/720 nm probe TA kinetics were fit using single exponential functions, which produced lifetimes of $\tau=30 \pm 1$ ps for violacein in ethylene glycol and $\tau=90 \pm 5$ ps for violacein in glycerol. In acetone, the violacein $720 \mathrm{~nm}$ signal decays with a time constant of 4.5 $\pm 0.2 \mathrm{ps}$, which is nearly as short as the lifetime measured in methanol, and the fluorescence quantum yield is similarly low.

Kinetics obtained with $570 \mathrm{~nm}$ probing, displayed in Figure $5 b$, exhibit two distinct time scales in ethylene glycol and glycerol. Biexponential fitting yielded lifetimes of $\tau=7 \pm 0.8$ ps 


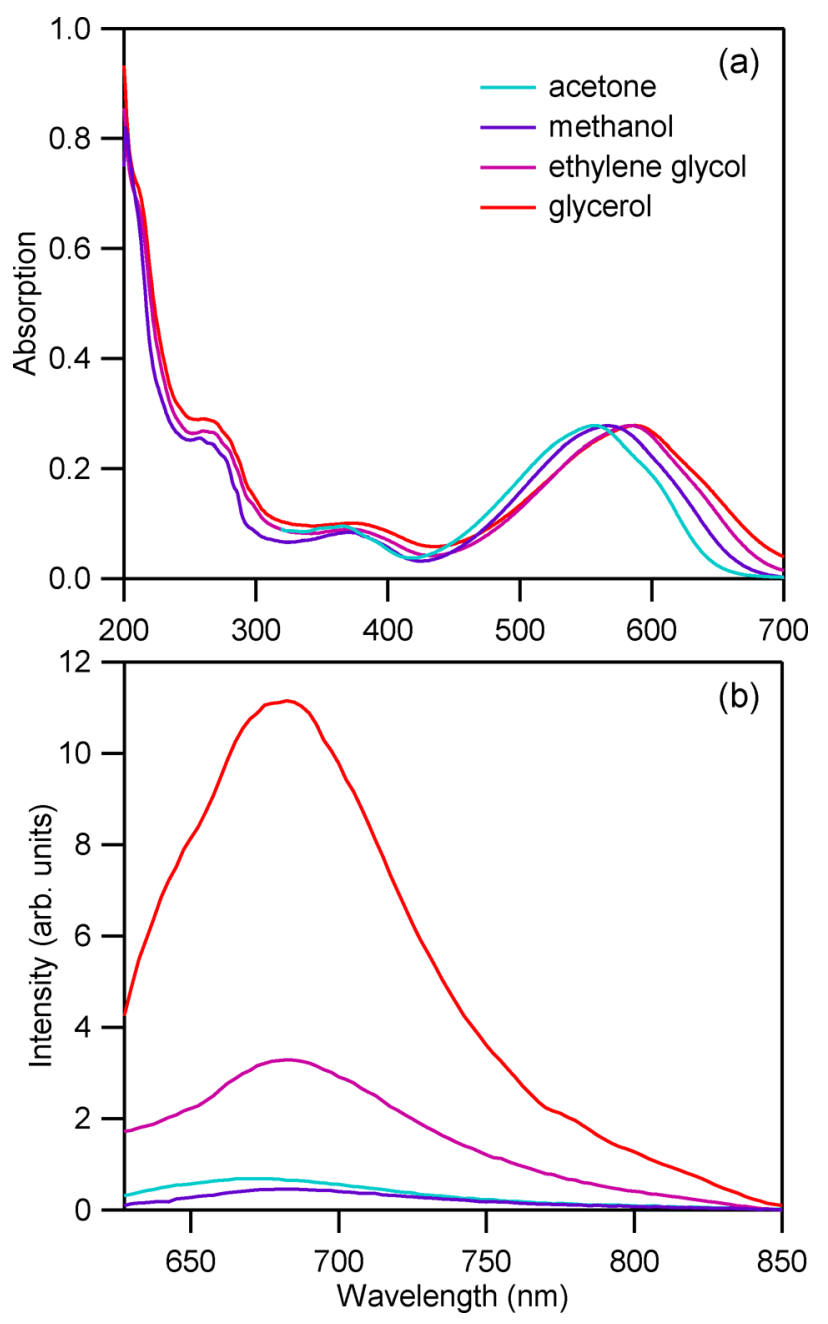

Figure 4. (a) UV-visible absorption and (b) steady-state fluorescence spectra of violacein in the solvents indicated upon $600 \mathrm{~nm}$ excitation. Note that the absorption spectra are scaled for comparison of the longest wavelength transition.

Table 2. Wavelengths of Maximum Absorption $\left(\lambda_{\max }^{\text {abs }}\right)$ and Emission $\left(\lambda_{\max }^{\mathrm{fl}}\right)$ and Fluorescence Quantum Yields $\left(\phi_{\mathrm{fl}}\right)$ of Violacein in Various Solvents at Room Temperature

\begin{tabular}{lccc} 
& $\lambda_{\max }^{\text {abs }}(\mathrm{nm})$ & $\lambda_{\max }^{\mathrm{fl}}(\mathrm{nm})$ & $\phi_{\mathrm{fl}} \times 10^{4}$ \\
\hline acetone & 556 & 668 & 2.0 \\
methanol & 569 & 680 & 1.4 \\
ethylene glycol & 584 & 683 & 10.8 \\
glycerol & 587 & 683 & 33.0 \\
\hline
\end{tabular}

and $\tau=52 \pm 3$ ps for violacein in ethylene glycol and $\tau=15 \pm$ 2 ps and $\tau=140 \pm 12$ ps for violacein in glycerol. In acetone, the $570 \mathrm{~nm}$ probe signal decays monoexponentially, similar to the $570 \mathrm{~nm}$ probe kinetics observed in methanol. The acetone kinetics at this probe wavelength were fit with a monoexponential function with a lifetime of $\tau=6.3 \pm 0.2 \mathrm{ps}$. The lifetimes and their associated uncertainties from TA measurements using $600 \mathrm{~nm}$ pump and 720 and $570 \mathrm{~nm}$ probe wavelengths for all solutions are compared in Table 3.

\section{DISCUSSION}

4.1. Violacein Excited-State Deactivation and Dynamics. On the one hand, the negative $\Delta A$ signals at $720 \mathrm{~nm}$ are

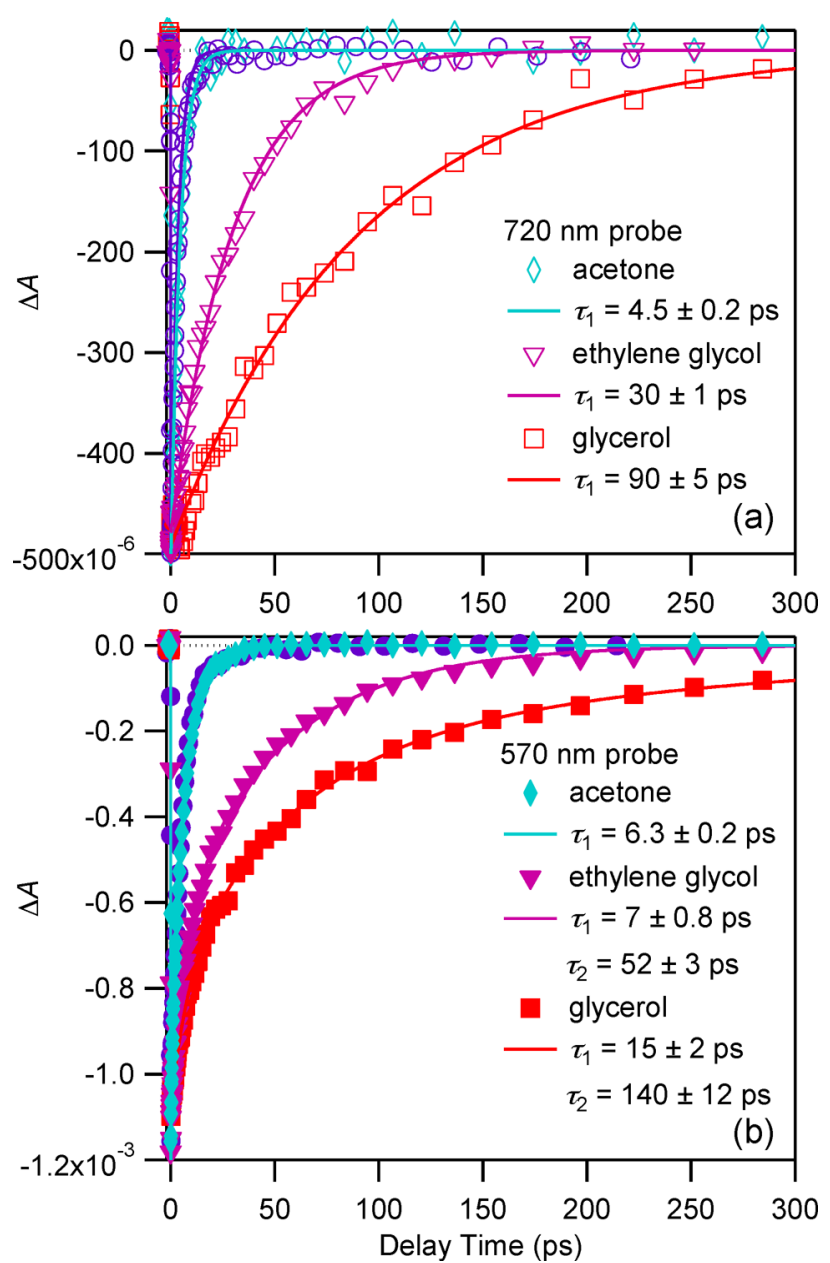

Figure 5. $600 \mathrm{~nm}$ pump TA kinetics of violacein in the solvents indicated collected with (a) 720 and (b) $570 \mathrm{~nm}$ probing. The kinetics of violacein in acetone were fit with a monoexponential function at both probe wavelengths. The kinetics in ethylene glycol and glycerol were fit with monoexponential functions at $720 \mathrm{~nm}$ and biexponential functions at $570 \mathrm{~nm}$. Note that the methanol kinetics are included in both panels for comparison.

Table 3. Kinetic Fit Parameters for the Stimulated Emission $(720 \mathrm{~nm})$ and Ground-State Bleach $(570 \mathrm{~nm})$ TA Signals of Violacein in the Solvents Listed

\begin{tabular}{lccc} 
& $\lambda_{\text {probe }}(\mathrm{nm})$ & $\tau_{1}(\mathrm{ps})$ & $\tau_{2}(\mathrm{ps})$ \\
acetone & 720 & $4.5 \pm 0.2$ & \\
\multirow{3}{*}{ methanol } & 570 & $6.3 \pm 0.2$ & \\
& 720 & $3.2 \pm 0.2$ & \\
ethylene glycol & 570 & $5.4 \pm 0.2$ & \\
& 720 & $30 \pm 1$ & \\
glycerol & 570 & $7 \pm 0.8$ & $52 \pm 3^{a}$ \\
& 720 & $90 \pm 5$ & \\
& 570 & $15 \pm 2$ & $140 \pm 12^{b}$
\end{tabular}

${ }^{a}$ The percent amplitudes of the $\tau_{1}$ and $\tau_{2}$ decay components are $35 \%$ and $65 \%$, respectively. ${ }^{b}$ The percent amplitudes of the $\tau_{1}$ and $\tau_{2}$ decay components are $48 \%$ and $52 \%$, respectively.

assigned to stimulated emission (SE), because this probe wavelength falls within the broad emission band observed from violacein between 600 and $850 \mathrm{~nm}$ (Figure 4b). On the other hand, the negative $\Delta A$ signals observed at $570 \mathrm{~nm}$ monitor ground-state repopulation, or ground-state bleaching (GSB), as 
this probe wavelength is close to the maximum of the longest wavelength absorption band (Figure 4a). From the SE kinetics measured at $720 \mathrm{~nm}$, violacein has an excited-state lifetime of $\sim 3$ ps in methanol (Figure 3a). The lifetime in acetone determined from the SE trace at $720 \mathrm{~nm}$ is only slightly longer. As the solvent viscosity increases, excited-state relaxation as measured by the stimulated emission lifetime at $720 \mathrm{~nm}$ slows significantly. Importantly, the measured fluorescence quantum yield increases proportionally (Tables 2 and 3 ). For example, as the excited-state lifetime increases from 3 to $90 \mathrm{ps}$ from methanol to glycerol, the fluorescence quantum yield also increases by a factor of $\sim 30$, from $1.4 \times 10^{-4}$ to $33 \times 10^{-4}$. This confirms that the excited state monitored by stimulated emission at $720 \mathrm{~nm}$ is responsible for the fluorescence observed in the steady-state experiments. Radiative lifetimes calculated by dividing the SE lifetime at $720 \mathrm{~nm}$ by the measured fluorescence quantum yield range between 22 and 30 ns. These values are reasonable for an organic fluorophore with the absorption strength of violacein.

Whenever internal conversion occurs on an ultrafast time scale of several picoseconds or less, the dissipation of excess vibrational energy from the hot ground state to the solvent can be the rate-limiting event that determines the kinetics of bleach recovery signals. ${ }^{30}$ In methanol and acetone, the observed GSB lifetimes are slightly longer than the SE lifetimes in the same solvents. The longer lifetimes are assigned to vibrational cooling dynamics. Similar rates of vibrational cooling have been observed for similarly sized solutes in these two solvents previously. ${ }^{31,32}$

The violacein excited-state lifetime increases in ethylene glycol and glycerol, which are more viscous than methanol, whereas the excited state decays on a similar time scale in acetone, which has a viscosity similar in magnitude to methanol. The solvent viscosity dependence suggests that a significant change in the molecular framework accompanies excited-state deactivation. Strong viscosity effects on excited-state lifetimes are typically encountered in molecular rotor dyes. ${ }^{33-35}$ The solvent viscosity-dependent fluorescence quantum yield of violacein may be indicative of a molecular rotor mechanism, as similar experimental observations have been made in stilbenes ${ }^{36}$ and cyanine dyes. ${ }^{37}$

If persistent photoproducts were formed, for example, as a result of isomerization, then the GSB signals would not recover to zero unless the photoproduct happened to have the same absorption cross section as the starting compound at the probe wavelength. Our data showing complete GSB recovery suggest that photoproduct formation is unimportant. Instead, we hypothesize that photoexcitation induces a significant change in molecular planarity that moves violacein toward a conical intersection between $S_{1}$ and $S_{0}$. This motion could involve cistrans isomerization about the $\mathrm{C}=\mathrm{C}$ double bond between the 2-PYR and OX moieties or torsional motion around the single bond between the 5-HI and 2-PYR ring systems. After deactivation through the $\mathrm{CI}$, the single bond torsion is reversed, and the molecule once again occupies its minimum energy nonplanar geometry. We hope that the observation of these ultrashort but exquisitely viscosity-dependent lifetimes will inspire computational studies that can elucidate the precise deactivation mechanism.

4.2. Photostability and Comparison with Other Natural Pigments. In methanol, virtually all excited-state violacein molecules return to the electronic ground state on a time scale of 3 ps. The fluorescence quantum yield in this solvent is $1.4 \times 10^{-4}$. The short excited-state lifetime and the low fluorescence quantum yield mimic the behavior of the canonical nucleobases, which are known to be highly photostable. ${ }^{38}$ Extremely efficient nonradiative decay from $S_{1}$ to $S_{0}$ prevents photochemical reactions from taking place. These characteristics combined with the absence of spectral changes after extended visible irradiation during the TA measurements suggest that violacein is photostable in methanol solution. This raises the possibility that violacein could play a photoprotective or sunscreening role in the bacteria it is found in. However, the increased excited-state lifetimes and fluorescence quantum yields observed here in viscous solvents suggest that violacein's photostability could be reduced whenever it is found in more viscous environments.

Konzen et al. showed that violacein can be incorporated into the lipid bilayer of Chromobacterium violaceum in high yields and that extraction of the pigment requires breaking of the cell walls. ${ }^{13}$ There is great interest in fluorescent probes that can measure viscosity in biological environments such as cell membranes. ${ }^{34,35}$ Interestingly, the viscosity-induced changes in the fluorescence lifetime of violacein, a natural membrane component, are comparable to ones observed with BODPY dyes that are among some of the most sensitive rotor dyes. ${ }^{33,35}$ Nevertheless, although emission by violacein is acutely sensitive to viscosity, its much lower fluorescence quantum yield makes it much less suitable as a viscosity probe. Future in situ measurements of violacein's photophysical properties in the bacteria it occurs in are needed to ascertain its effectiveness as a sunscreen.

Evaluating the photoprotective role of a bacterial secondary metabolite is not a trivial task. Not only should the molecule absorb solar radiation, its production must be induced upon irradiation, and it must provide effective screening and enhance the survivability of the organism under irradiation. ${ }^{39}$ Violacein meets the first two criteria, but the latter two properties are more difficult to establish. Oftentimes, the evidence in support of a molecule acting as a sunscreen is circumstantial. For instance, a major complication arises if the molecule in question also serves other vitally important physiological purposes. ${ }^{1}$ Violacein may increase bacterial survivability due to its antioxidative properties, ${ }^{13}$ and this active property complicates the determination of its efficacy as a passive sunscreen molecule. The difficulty inherent in designating a molecule as a sunscreen is the reason that of the many natural pigments found in biological systems (e.g., mycosporine-like amino acids, flavonoids in plants, melanin in humans, etc.), scytonemin produced in the sheaths of cyanobacteria is one of the only confirmed screening compounds. ${ }^{1}$

Like scytonemin and the carotenoids, violacein absorbs at visible wavelengths. ${ }^{2}$ The carotenoids zeaxanthin, astaxanthin, and staphyloxanthin were also isolated from bacteria in the Antarctic supraglacial streamwater samples. ${ }^{6}$ In solution, these compounds exhibit ultrafast excited-state deactivation, with lifetimes of 9 ps for zeaxanthin and 5 ps for astaxanthin. ${ }^{40,41}$ This is consistent with the characteristically low fluorescence of carotenoids. ${ }^{40}$ Whereas the carotenoids absorb most strongly between 350 and $500 \mathrm{~nm},{ }^{42,43}$ violacein has a broad absorption band that extends clear out to $700 \mathrm{~nm}$. It would be interesting to investigate whether the conversion of visible light energy to heat, which occurs with considerable efficiency in all solvents studied, would be beneficial for violacein-producing bacteria. 


\section{CONCLUSIONS}

A combined steady-state and time-resolved study of the excitedstate dynamics of violacein in methanol, and several other solvent environments, was presented for the first time. The fluorescence quantum yields in the low-viscosity solvents methanol and acetone are on the order of $1 \times 10^{-4}$, on par with the ultralow fluorescence quantum yields of the DNA/ RNA nucleobases. Excited state decay occurs on a time scale of $\sim 3$ ps in methanol. Strikingly, the excited-state lifetime increases in more viscous solvents such as ethylene glycol and glycerol, with concomitant increases in fluorescence quantum yield. We hypothesize that excited violacein decays nonradiatively via a conical intersection that is accessed by large amplitude motions. Further work is warranted to understand the in vivo photophysics of this pigment and the role it plays in providing photoprotection for bacteria.

\section{ASSOCIATED CONTENT}

\section{S Supporting Information}

The Supporting Information is available free of charge on the ACS Publications website at DOI: 10.1021/acs.jpcb.7b05769.

Details of the purification of violacein from bacterial cell components are given, including the characterization of the pigment samples used in spectroscopic measurements. The results of the $265 \mathrm{~nm}$ excitation TA experiments and absorption spectra recorded before and after TA measurements are also presented (PDF)

\section{AUTHOR INFORMATION}

\section{Corresponding Authors}

*E-mail: cforeman@montana.edu. Phone: +1 406-994-7361. (C.F.)

*E-mail: kohler.40@osu.edu. Phone: +1 614-688-2635. (B.K.)

ORCID $\odot$

Bern Kohler: 0000-0001-5353-1655

\section{Present Addresses}

${ }^{\perp}$ University Information Technology, PO Box 173240, Montana State University, Bozeman, MT 59717, USA.

\#Department of Biomedical Engineering, University of Minnesota, Minneapolis, Minnesota 55455, USA.

Notes

The authors declare no competing financial interest.

\section{ACKNOWLEDGMENTS}

This work was partially supported by National Aeronautics and Space Administration Grant No. NNX12AG77G and National Science Foundation Grant Nos. ANT-0839075 and ANT1141936. The mass spectrometry facility at MSU receives funding from the Murdock Charitable Trust and NIGMS NIH P20GM103474 of the IDEA program.

\section{REFERENCES}

(1) Cockell, C. S.; Knowland, J. Ultraviolet Radiation Screening Compounds. Biol. Rev. Cambridge Philos. Soc. 1999, 74, 311-345.

(2) Proteau, P. J.; Gerwick, W. H.; Garcia-Pichel, F.; Castenholz, R. The Structure of Scytonemin, an Ultraviolet Sunscreen Pigment from the Sheaths of Cyanobacteria. Experientia 1993, 49, 825-829.

(3) Castenholz, R. W.; Garcia-Pichel, F. Cyanobacterial Responses to UV-Radiation. In The Ecology of Cyanobacteria; Kluwer Academic Publishers: Dordrecht, The Netherlands, 2002; pp 591-611.

(4) Karsten, U.; Sawall, T.; Hanelt, D.; Bischof, K.; Figueroa, F. L.; Flores-Moya, A.; Wiencke, C. An Inventory of UV-Absorbing
Mycosporine-Like Amino Acids in Macroalgae from Polar to WarmTemperate Regions. Bot. Mar. 1998, 41, 443-453.

(5) Dunlap, W. C.; Shick, J. M.; Yamamoto, Y. Sunscreens, Oxidative Stress and Antioxidant Functions in Marine Organisms of the Great Barrier Reef. Redox Rep. 1999, 4, 301-306.

(6) Dieser, M.; Greenwood, M.; Foreman, C. M. Carotenoid Pigmentation in Antarctic Heterotrophic Bacteria as a Strategy to Withstand Environmental Stresses. Arct. Antarct. Alp. Res. 2010, 42, 396-405.

(7) Nienow, J. A.; McKay, C. P.; Friedmann, E. I. The Cryptoendolithic Microbial Environment in the Ross Desert of Antarctica - Light in the Photosynthetically Active Region. Microb. Ecol. 1988, 16, 271-289.

(8) Staehelin, J.; Harris, N. R. P.; Appenzeller, C.; Eberhard, J. Ozone Trends: A Review. Rev. Geophys. 2001, 39, 231-290.

(9) Foreman, C. M.; Cory, R. M.; Morris, C. E.; SanClements, M. D.; Smith, H. J.; Lisle, J. T.; Miller, P. L.; Chin, Y.-P.; McKnight, D. M. Microbial Growth under Humic-Free Conditions in a Supraglacial Stream System on the Cotton Glacier, Antarctica. Environ. Res. Lett. 2013, 8, 035022 .

(10) Smith, H. J.; Foreman, C. M.; Akiyama, T.; Franklin, M. J.; Devitt, N. P.; Ramaraj, T. Genome Sequence of Janthinobacterium Sp. Cg23_2, a Violacein-Producing Isolate from an Antarctic Supraglacial Stream. Genome Announce 2016, 4, e01468-15.

(11) Margalith, P. Z. Pigment Microbiology; Chapman and Hall: London, England, 1992.

(12) Mojib, N.; Farhoomand, A.; Andersen, D. T.; Bej, A. K. UV and Cold Tolerance of a Pigment-Producing Antarctic Janthinobacterium Sp Ant5-2. Extremophiles 2013, 17, 367-378.

(13) Konzen, M.; De Marco, D.; Cordova, C. A. S.; Vieira, T. O.; Antonio, R. V.; Creczynski-Pasa, T. B. Antioxidant Properties of Violacein: Possible Relation on Its Biological Function. Bioorg. Med. Chem. 2006, 14, 8307-8313.

(14) Cao, W.; Chen, W.; Sun, S.; Guo, P.; Song, J.; Tian, C. Investigating the Antioxidant Mechanism of Violacein by Density Functional Theory Method. J. Mol. Struct.: THEOCHEM 2007, 817, $1-4$

(15) Pickett, J. E.; Moore, J. E. Photodegradation of UV Screeners. Polym. Degrad. Stab. 1993, 42, 231-244.

(16) Beckstead, A. A.; Zhang, Y. Y.; de Vries, M. S.; Kohler, B. Life in the Light: Nucleic Acid Photoproperties as a Legacy of Chemical Evolution. Phys. Chem. Chem. Phys. 2016, 18, 24228-24238.

(17) Baker, L. A.; Greenough, S. E.; Stavros, V. G. A Perspective on the Ultrafast Photochemistry of Solution-Phase Sunscreen Molecules. J. Phys. Chem. Lett. 2016, 7, 4655-4665.

(18) Otterstedt, J.-E. A. Photostability and Molecular Structure. J. Chem. Phys. 1973, 58, 5716-5725.

(19) Chen, J.; Thazhathveetil, A. K.; Lewis, F. D.; Kohler, B. Ultrafast Excited-State Dynamics in Hexaethyleneglycol-Linked DNA Homoduplexes Made of A.T Base Pairs. J. Am. Chem. Soc. 2013, 135, 1029010293.

(20) Chen, J.; Kohler, B. Base Stacking in Adenosine Dimers Revealed by Femtosecond Transient Absorption Spectroscopy. J. Am. Chem. Soc. 2014, 136, 6362-6372.

(21) Rettori, D.; Duran, N. Production, Extraction and Purification of Violacein: An Antibiotic Pigment Produced by Chromobacterium Violaceum. World J. Microbiol. Biotechnol. 1998, 14, 685-688.

(22) Dias, L. C.; Da Motta Neto, J. D.; Rettori, D.; Duran, N. Semiempirical Indo/S Study on the Absorption Spectrum of Violacein. J. Mol. Struct:: THEOCHEM 2002, 580, 85-90.

(23) Wang, H.; Jiang, P.; Lu, Y.; Ruan, Z.; Jiang, R.; Xing, X.-H.; Lou, K.; Wei, D. Optimization of Culture Conditions for Violacein Production by a New Strain of Duganella Sp B2. Biochem. Eng. J. 2009, 44, 119-124.

(24) Mendes, A. S.; de Carvalho, J. E.; Duarte, M. C. T.; Duran, N.; Bruns, R. E. Factorial Design and Response Surface Optimization of Crude Violacein for Chromobacterium Violaceumi Production. Biotechnol. Lett. 2001, 23, 1963-1969. 
(25) Durán, N.; Justo, G. Z.; Ferreira, C. V.; Melo, P. S.; Cordi, L.; Martins, D. Violacein: Properties and Biological Activities. Biotechnol. Appl. Biochem. 2007, 48, 127-133.

(26) Hebert, P.; Baldacchino, G.; Gustavsson, T.; Mialocq, J. C. Photochemistry of an Unsymmetrical Polymethine-Cyanine Dye Solute Solvent Interactions and Relaxation Dynamics of LDS-751. J. Photochem. Photobiol., A 1994, 84, 45-55.

(27) Handbook of Chemistry and Physics, 84th ed.; Lide, D. R., Ed.;

CRC Press: Boca Raton, FL, 2004.

(28) Fogg, E. T.; Hixson, A. N.; Thompson, A. R. Densities and Refractive Indexes for Ethylene Glycol-Water Solutions. Anal. Chem. 1955, 27, 1609-1611.

(29) Hoyt, L. F. New Table of the Refractive Index of Pure Glycerol at 20 Degrees C. Ind. Eng. Chem. 1934, 26, 329-332.

(30) Middleton, C. T.; Cohen, B.; Kohler, B. Solvent and Solvent Isotope Effects on the Vibrational Cooling Dynamics of a DNA Base Derivative. J. Phys. Chem. A 2007, 111, 10460-10467.

(31) Terazima, M. Vibrational Relaxation from Electronically Photoexcited States in Solution Studied by the Acoustic Peak Delay Method: Hydrogen Bonding Effect to Betaine-30. Chem. Phys. Lett. 1999, 305, 189-196.

(32) Miyata, R.; Kimura, Y.; Terazima, M. Intermolecular Energy Transfer from the Photo-Excited Molecule to Solvent: Malachite Green. Chem. Phys. Lett. 2002, 365, 406-412.

(33) Vysniauskas, A.; Qurashi, M.; Gallop, N.; Balaz, M.; Anderson, H. L.; Kuimova, M. K. Unravelling the Effect of Temperature on Viscosity-Sensitive Fluorescent Molecular Rotors. Chem. Sci. 2015, 6, 5773-5778.

(34) Wu, Y.; Stefl, M.; Olzynska, A.; Hof, M.; Yahioglu, G.; Yip, P.; Casey, D. R; Ces, O.; Humpolickova, J.; Kuimova, M. K. Molecular Rheometry: Direct Determination of Viscosity in L-O and L-D Lipid Phases Via Fluorescence Lifetime Imaging. Phys. Chem. Chem. Phys. 2013, 15, 14986-14993.

(35) Kuimova, M. K. Mapping Viscosity in Cells Using Molecular Rotors. Phys. Chem. Chem. Phys. 2012, 14, 12671-12686.

(36) Sharafy, S.; Muszkat, K. A. Viscosity Dependence of Fluorescence Quantum Yields. J. Am. Chem. Soc. 1971, 93, 41194125.

(37) Murphy, S.; Sauerwein, B.; Drickamer, H. G.; Schuster, G. B. Spectroscopy of Cyanine Dyes in Fluid Solution at Atmospheric and High Pressure: The Effect of Viscosity on Nonradiative Processes. J. Phys. Chem. 1994, 98, 13476-13480.

(38) Onidas, D.; Markovitsi, D.; Marguet, S.; Sharonov, A.; Gustavsson, T. Fluorescence Properties of DNA Nucleosides and Nucleotides: A Refined Steady-State and Femtosecond Investigation. J. Phys. Chem. B 2002, 106, 11367-11374.

(39) Garciapichel, F.; Wingard, C. E.; Castenholz, R. W. Evidence Regarding the UV Sunscreen Role of a Mycosporine-Like Compound in the Cyanobacterium Gloeocapsa Sp. Appl. Environ. Microbiol. 1993, 59, $170-176$.

(40) Staleva, H.; Zeeshan, M.; Chabera, P.; Partali, V.; Sliwka, H.-R.; Polivka, T. Ultrafast Dynamics of Long Homologues of Carotenoid Zeaxanthin. J. Phys. Chem. A 2015, 119, 11304-11312.

(41) Ilagan, R. P.; Christensen, R. L.; Chapp, T. W.; Gibson, G. N.; Pascher, T.; Polivka, T.; Frank, H. A. Femtosecond Time-Resolved Absorption Spectroscopy of Astaxanthin in Solution and in AlphaCrustacyanin. J. Phys. Chem. A 2005, 109, 3120-3127.

(42) Asker, D.; Awad, T. S.; Beppu, T.; Ueda, K. Isolation, Characterization, and Diversity of Novel Radiotolerant CarotenoidProducing Bacteria. Methods Mol. Biol. (N. Y., NY, U. S.) 2012, 892, $21-60$.

(43) Clauditz, A.; Resch, A.; Wieland, K.-P.; Peschel, A.; Goetz, F. Staphyloxanthin Plays a Role in the Fitness of Staphylococcus Aureus and Its Ability to Cope with Oxidative Stress. Infect. Immun. 2006, 74, 4950-4953. 